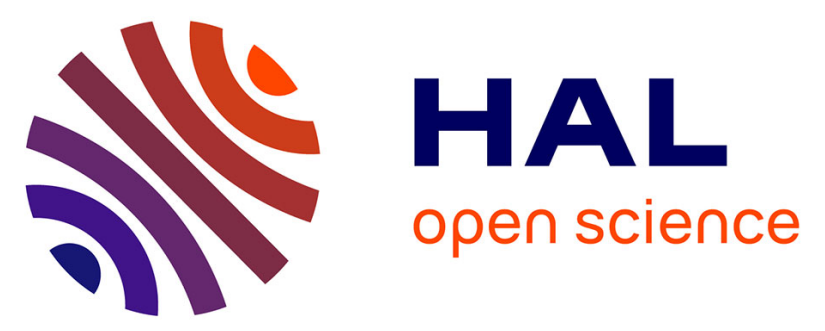

\title{
Le commerce international comme stratégie d'adaptation à la rareté des ressources hydriques? Utilité et application du concept de " commerce d'eau virtuelle " en Afrique du Nord
}

Nathalie Rousset

\section{To cite this version:}

Nathalie Rousset. Le commerce international comme stratégie d'adaptation à la rareté des ressources hydriques? Utilité et application du concept de " commerce d'eau virtuelle" en Afrique du Nord. Revue Tiers Monde, 2009, 199, pp.517-532. halshs-00435974

\section{HAL Id: halshs-00435974 \\ https://shs.hal.science/halshs-00435974}

Submitted on 25 Nov 2009

HAL is a multi-disciplinary open access archive for the deposit and dissemination of scientific research documents, whether they are published or not. The documents may come from teaching and research institutions in France or abroad, or from public or private research centers.
L'archive ouverte pluridisciplinaire HAL, est destinée au dépôt et à la diffusion de documents scientifiques de niveau recherche, publiés ou non, émanant des établissements d'enseignement et de recherche français ou étrangers, des laboratoires publics ou privés. 




LABORATOIRE D'ECONOMIE DE LA PRODUCTION

ET DE L'INTEGRATION INTERNATIONALE

UMR 5252 CNRS - UPMF

\section{CAHIER DE RECHERCHE \\ $\mathrm{N}^{\circ} 26$}

Le commerce international comme stratégie d'adaptation à la rareté des ressources hydriques ?

Utilité et application du concept de " commerce d'eau virtuelle " en Afrique du Nord.

(version augmentée et mise à jour de la note de travail LEPII n²4/2007)

\section{Nathalie Rousset}

2009

LEPII 


\title{
Le commerce international comme stratégie d'adaptation
}

\section{à la rareté des ressources hydriques?}

\section{Utilité et application du concept de " commerce d'eau virtuelle " en Afrique du Nord}

Nathalie Rousset,

LEPII, Université de Grenoble, CNRS ; nathalie.rousset@upmf-grenoble.fr

Mise à jour 2009

\begin{abstract}
Résumé : Le changement climatique amplifie les risques pesant sur les ressources hydriques et les capacités agricoles au sud de la Méditerranée. Cet article analyse la portée méthodologique du concept de "commerce d'eau virtuelle » en quantifiant le contenu en eau des importations céréalières des pays d'Afrique du Nord. Il montre aussi sa pertinence en tant qu'outil stratégique, susceptible de structurer les politiques d'adaptation à la rareté croissante de l'eau. Le remodelage sectoriel de l'allocation des ressources que permettrait l'importation des produits intensifs en eau pourrait être une mesure holistique d'adaptation à la rareté mais dont l'opportunité reste tributaire de la configuration des accords commerciaux.
\end{abstract}

Mots-clés : commerce d'eau virtuelle, changement climatique, agriculture, Afrique du Nord

\section{Introduction}

L'eau est un enjeu majeur pour le développement durable en Afrique du Nord, et les usages de la ressource sont au centre de la problématique. Le déséquilibre entre les ressources et les besoins agricoles devrait sans doute s'aggraver durant les prochaines décennies. Les prospectives sur le changement climatique et la démographie montrent des besoins croissants, mais des disponibilités hydriques et les rendements agricoles seront plus restreints que prévus.

Face aux difficultés des cultures céréalières et aux risques de tentatives d'un recours accru à l'irrigation pour y pallier, cet article pose la question du rôle du commerce d'eau virtuelle dans la définition de stratégies d'adaptation à la rareté structurelle de l'eau en Afrique du Nord. Ce concept introduit par Allan (1996) décrit les volumes d'eau incorporés virtuellement dans la production des biens échangés sur les marchés internationaux. S'il a surtout été développé dans une perspective géopolitique pour caractériser la gestion des risques de conflits sur la ressource au Moyen-Orient, l’objectif ici est de dépasser cette approche pour analyser sa dimension économique et examiner sa pertinence en tant qu'instrument de gestion des déficits hydriques.

Au-delà de la dimension incompressible des importations alimentaires et de l'utilisation ad hoc de l'eau virtuelle, l'utilisation stratégique de cet instrument, par le déplacement des capacités d'irrigation allouées aux céréales vers des secteurs à haute valeur ajoutée et l'importation des produits alimentaires de base, pourrait être une mesure holistique de gestion de la rareté de l'eau en Afrique du Nord. La première partie met en relief les risques du changement climatique sur les ressources hydriques et l'agriculture et pose la question de l'importation d'eau virtuelle comme alternative à l'accroissement des usages céréaliers de l'eau. On aborde ensuite la dimension méthodologique de ce concept en quantifiant les volumes d'eau virtuelle importés sous forme de blé par l'Egypte, l'Algérie et le Maroc afin de caractériser son importance pour pallier les déficits hydriques. On discute enfin de l'opportunité et des risques d'une utilisation stratégique de cet outil pour remodeler l'allocation sectorielle de la ressource dans la région.

\section{1 - L'eau et l'agriculture au défi du changement climatique en Afrique du Nord}

\section{1- 1 Prospectives sur les impacts du changement climatique}

Les paysages agricoles d'Afrique du Nord ont pour caractéristiques communes d’être limités par 
les ressources hydriques et de présenter une forte vulnérabilité à la variabilité climatique. Les simulations du modèle MAGICC ${ }^{1}$ (Hulme et al, 2000), centrées sur la région, estiment un réchauffement de $1^{\circ} \mathrm{C}$ entre 1990 et 2030 et une tendance à la baisse de la pluviométrie, de l'ordre de 5 à $10 \%$. D'ici 2050, les températures pourraient augmenter de $3^{\circ} \mathrm{C}$ et les précipitations diminuer de 10 à $30 \%$ (Boko et al, 2007). Des changements dans les moyennes, mêmes faibles, impliquent un accroissement des extrêmes climatiques (Katz et Brown, 1992). La probable augmentation de la fréquence et de l'intensité des sécheresses, auxquelles les systèmes hydriques et agricoles sont particulièrement sensibles, est le risque principal dans la région. Les conditions défavorables de ces dernières décennies pourraient ainsi devenir progressivement la norme au sud de la Méditerranée.

La gestion de l'eau est un problème récurrent et qui conditionne l'avenir de la région. La forte sensibilité des bassins hydrologiques maghrébins à de faibles écarts climatiques implique que les disponibilités seront fortement touchées par la diminution du ruissellement ${ }^{2}$ (Agoumi et al., 1999 ; PNUD-FEM, 1998). Le changement climatique placerait ainsi la région dans une situation inconfortable puisque les volumes mobilisables seront à la limite des besoins sectoriels, voire déficitaires en Algérie et en Egypte en 2020.

Les modèles agroclimatiques montrent que l'accélération d'une dynamique d'accroissement des déficits hydriques entraînera une baisse des rendements des principales cultures. En 2030, le changement climatique pourrait amputer les rendements du blé de $10 \%$ en Algérie et de $20 \%$ en Egypte et au Maroc (Bindi et Moriondo, 2005). La vulnérabilité des céréales à la variabilité des précipitations, déjà manifeste au Maghreb, montre que les risques dépendent surtout de l'accentuation attendue des sécheresses. L'amplification des fluctuations de la production depuis les années 1980, en réponse à la baisse tendancielle des précipitations et à l'augmentation des sécheresses, sert d'analogue pour anticiper les risques du changement climatique. Cette sensibilité se traduit par des épisodes de contraction et d'expansion de l'activité, aux effets macroéconomiques déstabilisateurs. Les impacts des fluctuations agricoles sur le PIB ou l'emploi, et les chocs qu'elles génèrent en termes de recours aux importations céréalières, sont particulièrement dommageables au développement.

\section{1- 2 Stratégies d'adaptation à la rareté de la ressource}

Le changement climatique exacerbera des problèmes existants de pression sur les ressources et de productions agricoles en difficulté. La réduction mondiale des émissions de GES peut réduire l'ampleur du changement climatique à long terme, mais l'adaptation est incontournable pour contenir les dommages attendus dès le court terme. En Afrique du Nord, l'adaptation à la rareté croissante de l'eau est un enjeu majeur, d'autant qu'elle réduirait ces risques futurs tout en limitant les dommages déjà coûteux des sécheresses.

Les mesures d'atténuation des impacts sur les rendements céréaliers concernent essentiellement les ressources hydriques et l'irrigation. Il s'agirait d'accélérer la mobilisation de ressources conventionnelles avec de nouveaux barrages, et surtout le développement de capacités non conventionnelles, par le dessalement de l'eau de mer pour l'approvisionnement des villes côtières et la redistribution de l'eau des barrages vers les cultures, ou la réutilisation agricole des eaux usées. Au-delà de ces options d'offre, les économies potentielles sur les besoins sectoriels,

\footnotetext{
${ }^{1}$ MAGICC sert de référence pour les projections de l'IPCC (Intergovernmental Panel on Climate Change). Nos simulations pour l'Afrique du Nord sont basées sur le scénario d'émission de GES P50, trajectoire médiane des scénarios décrits par Nakicenovic et al (2000), conduisant à un forçage radiatif proche d'une stabilisation des concentrations de $\mathrm{CO}_{2}$ à 650 ppm.

${ }^{2}$ Pour les bassins versants marocains, les modèles hydrologiques anticipent une réduction des écoulements de surface de 10 à $15 \%$ pour un réchauffement de $1^{\circ} \mathrm{C}$ et une baisse de la pluviométrie de 0 à $5 \%$ (Senouci, 2002). En Algérie, les déficits des apports en eaux superficielles s'élèveraient à 15 et $30 \%$ pour des réchauffements de 0,5 et $1^{\circ} \mathrm{C}$ et des réductions des précipitations de 10 et $15 \%$ (Ministère de l'Aménagement du Territoire et de l'Environnement, 2001).
} 
générées par une Gestion Intégrée des Ressources en Eau³, seront probablement le principal gisement qu'il sera possible de dégager (Benblidia et al, 1997). Cette approche pose cependant la question des difficultés et des risques d'un accroissement des usages agricoles de l'eau. Dans le contexte de croissance démographique et des prospectives de développement économique dans lequel s'inscrit le changement climatique, tous les secteurs verront leurs besoins s'accroître. Dans quelle mesure la redistribution intersectorielle de l'eau sera donc suffisante pour satisfaire les besoins des cultures et pallier les effets du changement climatique, pour quelle efficacité, et pour combien de temps?

Les politiques agricoles ont conduit à une concentration sectorielle des disponibilités hydriques. L'efficacité d'une amélioration de l'accès à l'eau des céréales en Afrique du Nord et la durabilité de cette stratégie est très relative puisqu'elle ne permettrait pas d'enrayer la dépendance structurelle aux importations et serait rapidement limitée par l'évolution des volumes mobilisables. Le poids de l'irrigation est aussi un enjeu sensible dans la mesure où il constitue un handicap au développement d'autres secteurs. Les conflits intersectoriels déjà intenses sur les usages et l'évolution attendue des disponibilités et des besoins montrent que les possibilités d'étendre l’irrigation seront très limitées (Margat et Vallée, 1999).

Les enjeux de l'adaptation à la rareté de l'eau dépassent les seuls impacts du changement climatique sur les rendements agricoles et doivent voir au-delà de mesures palliatives pour les soutenir. Pour être robuste, elle doit être appréhendée à l'aune des problématiques plus larges posées par la promotion du développement durable. En Afrique du Nord elle doit être analysée au spectre des politiques de gestion des ressources hydriques, en intégrant notamment la question des usages sectoriels alternatifs.

L'inadaptabilité croissante des céréales à l'environnement bioclimatique et le coût pour les autres secteurs d'une fuite en avant que constituerait une tentative d'accroissement des usages céréaliers de l'eau montrent que la réduction de la vulnérabilité pourrait passer par la promotion d'activités moins dépendantes des ressources hydriques et l'importation des produits intensifs en eau comme les céréales. L'eau virtuelle se profile ainsi comme un outil d'allègement des contraintes hydriques, alternatif au recours accru à l'irrigation. Le commerce agricole serait une source alternative d'eau, dont l'utilisation stratégique pourrait devenir attractive pour améliorer la sécurité hydrique et remodeler les termes de l'allocation sectorielle des ressources domestiques. En dépit du poids de l'irrigation, cet outil est déjà incontournable pour pallier les déficits céréaliers.

\section{2 - La mesure du commerce d'eau virtuelle en Afrique du Nord}

Le concept de commerce d'eau virtuelle traduit le contenu en eau du commerce international. Le passage à une définition quantitative, qui permette de mesurer les flux associés aux importations agricoles, peut être conduit selon deux approches. La première reflète le point de vue de l'offre et analyse les volumes d'eau engagés dans le processus productif du pays exportateur. La seconde, plus pertinente pour les pays caractérisés par des déficits hydriques structurels, se base sur les conditions de production de l'importateur pour estimer les ressources domestiques économisées par les importations de produits intensifs en eau.

\section{2- 1 Quantification des importations d'eau virtuelle}

\footnotetext{
${ }^{3}$ La GIRE repose sur des principes définis lors de la Conférence des Nations unies sur l'eau et l'environnement (Dublin, 1992) traduit selon lesquels les déficits hydriques sont d'abord la conséquence de déficits de gestion et de gouvernance de la ressource. Elle s'oppose aux approches sectorielles et fondées sur l'offre qui prévalent depuis les années 1960, et s'appui sur la gestion intersectorielle de la demande et de la ressource à l'échelle du bassin versant.
} 
La mesure traditionnelle de l'eau virtuelle évalue les volumes d'eau utilisés pour la production des biens importés selon les conditions de l'offre. On a calculé les importations d'eau virtuelle de l'Algérie, de l'Egypte et du Maroc entre 1997 et 2005 en se focalisant sur le blé, principale céréale importée. La méthodologie ${ }^{4}$ suivie évalue les flux effectifs d'eau virtuelle en se basant sur les quantités consommées selon l'origine des importations. Les estimations se fondent sur les bases de données des échanges agricoles (FAO, TradeSTAT) et sur les estimations des Demandes Spécifiques en Eau (DSE) de la production de blé des pays exportateurs (Hoekstra et Hung, 2002).

Tableau 1 : Importations d’eau virtuelle sous forme de blé (1997-2005)

\begin{tabular}{cccc}
\hline & $\begin{array}{c}\text { Volume cumulé sur } \\
\text { la période }\left(\text { millions } \mathbf{~ d e ~}^{3}\right)\end{array}$ & $\begin{array}{c}\text { Volume annuel } \\
\text { moyen }\left(\mathbf{m i l l i o n s ~} \mathbf{~ d e ~}^{3}\right)\end{array}$ & $\begin{array}{c}\text { Intensité hydrique } \\
\text { moyenne }\left(\mathbf{m}^{3} / \text { tonne }\right)\end{array}$ \\
\hline Egypte & 69280 & 7698 & 1475 \\
Algérie & 52492 & 5832 & 1230 \\
Maroc & 28164 & 3129 & 1133 \\
\hline Total & 149946 & 16660 & 1280 \\
\hline
\end{tabular}

Sources : Calculs d’après FAO, TradeSTAT et Hoekstra et Hung (2002)

Graphique 1 : Evolution des importations d'eau virtuelle sous forme de blé (1997-2005)

importation d'eau virtuelle

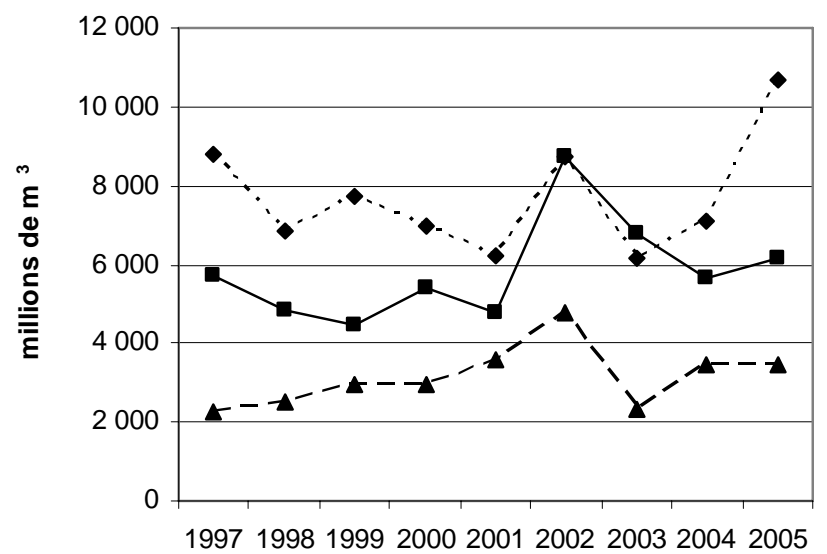

intensité hydrique des importations

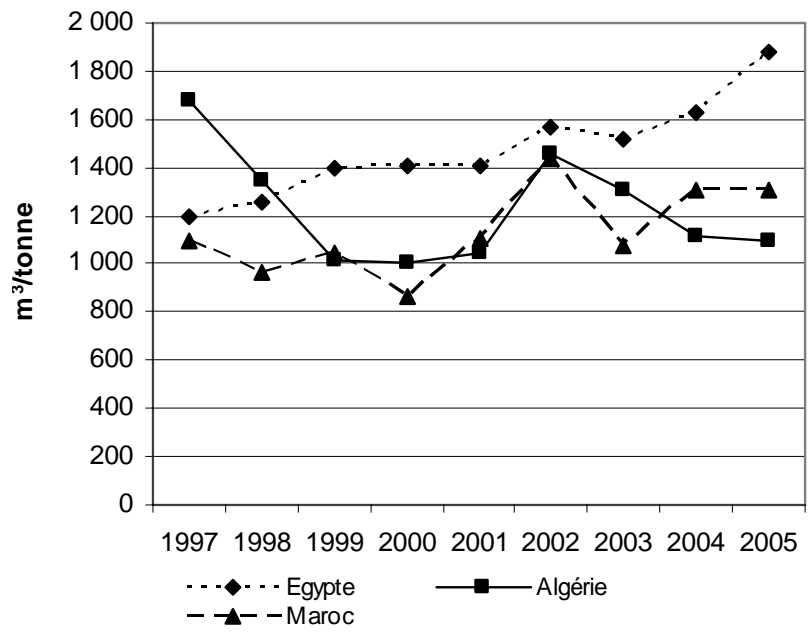

Sources : ibid.

L’importance des volumes d'eau importés par le biais du commerce du blé, avoisinant les 150 $\mathrm{Gm}^{3}$ sur la période, est le premier aspect qui se dégage des résultats reportés dans le tableau et le graphique 1. Les contrastes régionaux sont significatifs et l'Egypte occupe systématiquement le haut du tableau en cumulant les plus fortes importations en volume et l'intensité hydrique des

\footnotetext{
${ }^{4}$ L'estimation des flux d'eau virtuelle se fonde sur le concept de DSE ( $\mathrm{m}^{3} /$ tonnes), défini comme le ratio entre les besoins spécifiques en eau $\left(\mathrm{m}^{3} / \mathrm{ha}\right.$ ) et les rendements (tonne/ha), par culture et par pays. Les volumes d'eau importés par le biais du commerce du blé sont obtenus par le produit des volumes de blé importés et de leur contenu en eau, tel que CEV $\left[n_{e}, n_{m}, c, t\right]=E C$ $\left[n_{e}, n_{m}, c, t\right] \times$ DSE $\left[n_{e}, c\right]$; avec CEV les flux d'eau virtuelle $\left(m^{3} / a n\right)$ du pays exportateur $n_{e}$ vers le pays importateur $n_{m}$ en conséquence du commerce du produit c, ici le blé. EC représente les flux de commerce en volume (tonnes/an) du pays exportateur $\mathrm{n}_{\mathrm{e}}$ vers le pays importateur $\mathrm{n}_{\mathrm{m}}$, durant l'année t et fonction de la DSE du blé du pays exportateur $\mathrm{n}_{\mathrm{e}}$.
} 
importations la plus élevée. Les conditions de production de ses partenaires commerciaux montrent que chaque tonne importée a un contenu en eau $30 \%$ supérieur à ce que l'on observe au Maroc. L’Egypte concentre ainsi près de la moitié des importations d'eau virtuelle de la région, avec des volumes annuels de l'ordre de $8 \mathrm{Gm}^{3}$.

Depuis 2000, l'Algérie suit de près les tendances égyptiennes et les écarts traduisent essentiellement les différences d'intensité hydrique de leurs fournisseurs respectifs. Le Maroc, très en dessous des niveaux atteints par ses voisins, enregistre néanmoins la plus forte augmentation sur la période, avec une hausse des importations d'eau virtuelle de $55 \%$, contre $21 \%$ en Egypte, qui marque pourtant la croissance la plus marquée de l'intensité hydrique des importations. En Algérie la progression n’a été que de $9 \%$, car l'augmentation massive des besoins en blé a été compensée par une forte réduction de leur contenu en eau ${ }^{5}$.

La hausse tendancielle des importations d'eau virtuelle se caractérise par d'importants pics annuels. En 2002, elles ont augmenté de 83 \% en Algérie, et de 41 et 33 \% en Egypte et au Maroc. Elles sont ainsi directement connectées aux fluctuations des déficits céréaliers générées par les sécheresses.

\section{2-2 Evaluation des ressources hydriques économisées en Afrique du Nord}

En adoptant le point de vue du consommateur, l'eau virtuelle caractérise les volumes qui auraient été nécessaires pour produire les céréales importées là où elles sont consommées. Cette approche traduit plus fidèlement le concept de commerce d'eau virtuelle puisque «c'est justement dans le passage du site de production au site de consommation que l'eau abandonne son statut réel pour son statut virtuel » (Renault, 2003). Pour les pays limités par leurs dotations, la valeur de l'eau incorporée dans les importations dépend moins des conditions d'utilisation de la ressource de leurs fournisseurs que de leurs propres contraintes hydriques et des volumes qui auraient dû être mobilisées sur place pour satisfaire les besoins alimentaires ${ }^{6}$.

Cette définition est utile pour l'analyse du coût d'opportunité des usages agricoles de l'eau. En quantifiant l'eau virtuelle comme les volumes économisés par les importations, étant donné les conditions locales d'utilisation des ressources, les résultats traduisent plus systématiquement l'insuffisance structurelle de l'eau, là où la question de produire ou d'importer les céréales se pose. Elle confère ainsi une position centrale à ce concept pour l'analyse des stratégies d'adaptation là où l'eau est rare.

Les estimations ${ }^{7}$ des importations d'eau virtuelle basées sur les DSE du blé en Afrique du Nord dépeignent un tableau éloigné de celles fondées sur les conditions d'offre. Les volumes en jeu, résumés dans le tableau et le graphique 2, sont sensiblement augmentés et la situation relative des pays de la région est inversée. Entre 1997 et 2005, le commerce international du blé a permis d'économiser près de $52 \mathrm{Gm}^{3} / \mathrm{an}$, les apports hydriques sont donc trois fois plus importants que ceux réellement importés, et ce résultat masque d'importants contrastes régionaux. Les volumes importés par l'Egypte sont en effet très inférieurs à l'Algérie, et dans une moindre mesure au Maroc. Toute la région enregistre de fortes progressions de ces apports en eau, mais les écarts se creusent puisqu'entre 1990 et 2005, la hausse approche $120 \%$ en Algérie, contre 80 et $60 \%$ au Maroc et en Egypte.

\footnotetext{
${ }^{5}$ Entre 1997 et 2005 les importations de blé ont augmenté de $66 \%$ en Algérie, mais leur intensité hydrique a diminué de $35 \%$ du fait de la substitution du Canada par la France dans le rôle de fournisseur principal.

${ }^{6}$ Les écarts entre les estimations des deux méthodes reflètent les volumes économisés au niveau mondial par la localisation de la production céréalière dans les pays qui présentent une productivité de l'usage de la ressource supérieure au pays importateur.

${ }^{7}$ Les importations d'eau virtuelle, selon l'approche par la demande, sont telles que CEV $\left[n_{e}, n_{m}, c, t\right]=E C\left[n_{e}, n_{m}, c, t\right] \times$ DSE $\left[n_{m}\right.$, c].
} 
Tableau 2 : Importations d'eau virtuelle sous forme de blé selon les volumes économisés (1997-2005)

\begin{tabular}{cccc}
\hline & $\begin{array}{c}\text { Volume cumulé sur } \\
\text { la période }\left(\text { millions de } \mathbf{~ m}^{3}\right)\end{array}$ & $\begin{array}{c}\text { Volume annuel } \\
\left.\text { moyen (millions de } \mathbf{~ m}^{3}\right)\end{array}$ & $\begin{array}{c}\text { Intensité hydrique } \\
\text { moyenne }\left(\mathbf{m}^{3} / \text { tonne) }\right.\end{array}$ \\
\hline Egypte & 48753 & 5417 & 1005 \\
Algérie & 311690 & 34632 & 7222 \\
Maroc & 103765 & 11529 & 3134 \\
\hline Total & 464208 & 51579 & 4120 \\
\hline
\end{tabular}

Sources : Calculs d’après FAO, TradeSTAT et Hoekstra et Hung (2002)

Graphique 2 : Evolution des volumes d’eau virtuelle économisés par les importations de blé (1990-2005)

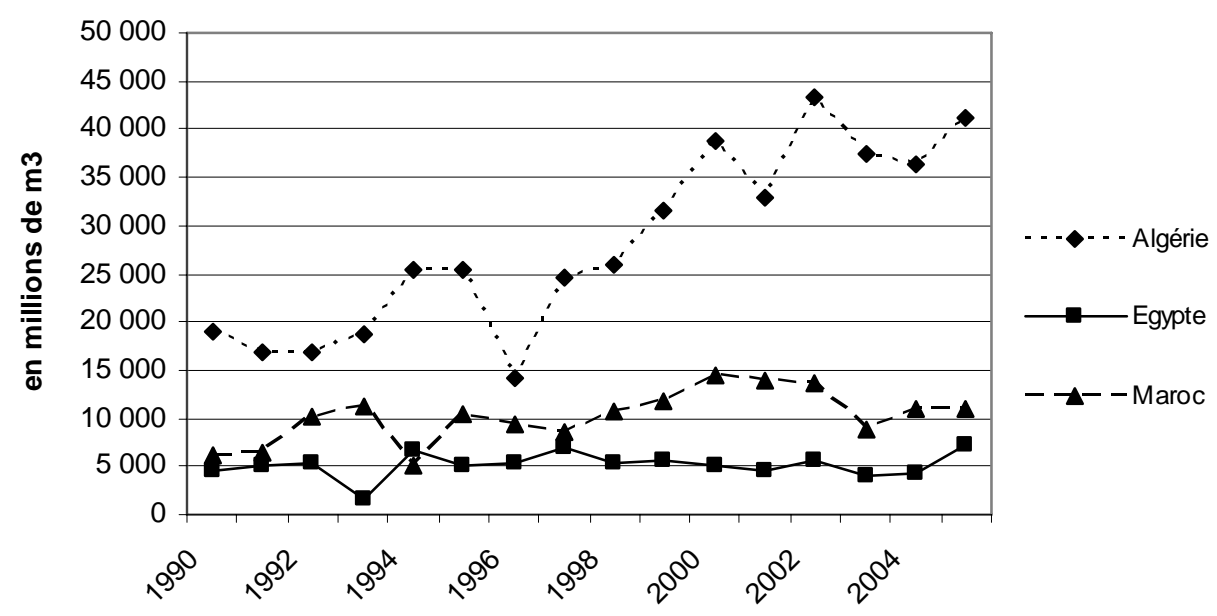

Sources : ibid.

En Algérie, le commerce du blé constitue un mode d'approvisionnement en eau essentiel avec une contribution de près de $35 \mathrm{Gm}^{3} / \mathrm{an}$. L'écart avec les calculs précédents traduit l'important différentiel entre l'intensité hydrique de la production de blé domestique et celle de ses fournisseurs. Ces importations participent ainsi indirectement à l'efficacité globale des usages de la ressource en économisant $29 \mathrm{Gm}^{3} / \mathrm{an}$ au niveau mondial. Son poids se mesure également au niveau régional puisque près de $70 \%$ de l'eau économisée s’y concentre. Ramenée à la population et comparée aux ressources domestiques, l'importance de ces apports hydriques est sans équivoque. L'eau incorporée dans ces flux de commerce représente près de 1250 $\mathrm{m}^{3} / \mathrm{hab}$./an, soit trois fois plus que les estimations des ressources hydriques renouvelables par habitant et six fois plus que les disponibilités domestiques effectives (FAO, AQUASTAT).

En Egypte les résultats sont inversés, puisque c'est le pays qui importe le plus massivement d'eau du point de vue de l'offre, mais également celui qui en économise très largement le moins. En important du blé de pays ayant une intensité hydrique supérieure aux conditions domestiques, les importations lui permettent d'économiser 5,4 $\mathrm{Gm}^{3}$ /an, mais résultent au niveau global en une perte nette de $2,5 \mathrm{Gm}^{3}$. Le commerce du blé se traduit donc par des économies nettement moindres que chez ses voisins et qui réduisent l'efficacité globale de l'utilisation de la ressource.

La gestion de l'eau revêt ici un fort contenu géopolitique car les ressources hydriques sont quasi exclusivement dépendantes des prélèvements du Nil, donc de ressources extérieures dont les 
volumes annuels sont fixés. Le commerce du blé permet de pallier la rigidité d'une ressource déjà surexploitée et d'économiser chaque année près de $10 \%$ sur les volumes alloués par l'accord de partage avec le Soudan. D'autre part, les importations de blé égyptiennes économisent sur l'irrigation, la production dépendant totalement du Nil. L'eau d'irrigation et l'eau issue des précipitations, diffèrent fondamentalement en termes de coût d'opportunité puisque seule la première peut être allouée à des usages alternatifs lorsque la production est remplacée par des importations.

Les estimations de l'eau économisée par les importations céréalières illustrent l'importance du commerce d'eau virtuelle puisque ces apports pourraient difficilement être substitués par des ressources domestiques. Le commerce joue donc un rôle crucial de redistribution internationale de l'eau, et apparaît incontournable pour substituer les besoins hydriques domestiques et atteindre la sécurité alimentaire dans la région. L’Algérie et l'Egypte, en particulier, mettent déjà en œuvre des stratégies ad hoc d'importation d'eau virtuelle pour pallier l'insuffisance des ressources.

\subsection{Prospective sur les importations d'eau virtuelle}

D’ici 2020, les besoins céréaliers vont croître rapidement avec l'évolution démographique, en Algérie en particulier, qui doit enregistrer une hausse de la demande de plus de $60 \%$, mais également en Egypte et au Maroc où les besoins augmenteront de $40 \%$ (Yang et Zehnder, 2002). Le poids des importations est déterminant malgré les contrastes régionaux. En supposant une stabilité de la composition de l'offre, les volumes importés seront dominés par l'Egypte qui avoisineront 14 millions de tonnes/an, pour des volumes de l'ordre de 10 et 6 millions de tonnes en Algérie et au Maroc.

Les importations de blé permettront ainsi d'économiser plus de $105 \mathrm{Gm}^{3}$ /an dans la région, dont $72 \mathrm{Gm}^{3}$ en Algérie, soit un doublement par rapport au début des années 2000. Aussi élevées soient-elles, ces projections sous-estiment l'évolution de la dépendance céréalière et hydrique, les taux d'autosuffisance diminuant de manière tendancielle depuis 30 ans. Avec l'intensification du stress hydrique générée par le changement climatique, les productions domestiques seront de plus en plus sous pression et verront leur capacité à répondre aux besoins croissants des populations se réduire.

L'articulation quantitative entre les disponibilités hydriques et les importations céréalières permet d'appréhender l'évolution du poids des importations dans les apports alimentaires. Les résultats de Yang et al (2003) mettent en évidence une relation entre la faiblesse des dotations hydriques et les importations céréalières par tête, et estiment un seuil de $1500 \mathrm{~m}^{3} / \mathrm{an} / \mathrm{hab}$. en dessous duquel elles croissent de manière exponentielle. En incluant d'autres facteurs comme le PIB et les terres arables, la relation est encore plus significative et décrit l'évolution nordafricaine, qui voit simultanément les capacités hydriques décroître avec la progression démographique, l'importation de volumes croissants de céréales, et une hausse sensible de la part des besoins alimentaires satisfaite par les importations. Durant les prochaines décennies, la chute des disponibilités en eau causée par les tendances démographiques et intensifiée par le changement climatique va donc accélérer une dynamique en cours de hausse des importations d'eau virtuelle pour satisfaire les besoins alimentaires.

\section{3 - L'importation d'eau virtuelle comme stratégie d'adaptation à la rareté de l'eau ?}

L'évaluation du contenu en eau des importations de blé illustre le poids structurel du commerce d'eau virtuelle pour la sécurité alimentaire et la gestion des déficits hydriques des pays limités par les ressources domestiques. Le commerce d'eau virtuelle, "économiquement invisible et 
politiquement silencieux » (Allan, 2001) est donc une réalité palpable en Afrique du Nord pour répondre à des contraintes internes fortes. Ces résultats poussent à dépasser la dimension méthodologique de ce concept pour analyser sa pertinence en tant qu'instrument, susceptible de structurer les politiques commerciales et agricoles, là où l'eau est rare. L'analyse du commerce d'eau virtuelle comme outil d'adaptation à la rareté croissante de l'eau nécessite d'aller au-delà des besoins ad hoc d'importation d'eau virtuelle, pour évaluer son opportunité en tant que vecteur potentiel d'un remodelage de l'allocation sectorielle de la ressource.

\subsection{Des besoins incompressibles aux stratégies d'allocation des ressources disponibles}

L'importation croissante d'eau virtuelle est incontournable pour gérer la progression des besoins céréaliers en Afrique du Nord. Il faut néanmoins distinguer les enjeux de natures différentes posés par les volumes incompressibles, qu'il faudra nécessairement importer face à l'insuffisance absolue des ressources hydriques domestiques, des stratégies d'allocation des ressources disponibles.

Le premier volet a une signification géoéconomique puisque l'utilisation du commerce international permet de pallier l'impossibilité de satisfaire les besoins par les productions domestiques. Du point de vue céréalier, la région est entrée dans une dynamique malthusienne où l'eau mobilisable est insuffisante pour assurer les besoins alimentaires de populations en forte croissance (Allan, 1996). Le faible poids du blé dans les importations en valeur et les substitutions importantes qu'elles permettent en termes d'usage de l'eau, suggèrent que l'importation des céréales constitue un moyen de gestion de la rareté absolue de la ressource économiquement viable dans la région.

A un second niveau, c'est l'efficacité de l'utilisation sectorielle de l'eau disponible qui est posée. Ce volet aborde la dimension économique des stratégies d'eau virtuelle, en tant qu'application élargie de la théorie des avantages comparatifs. Pour les pays subissant des déficits structurels, les importations d'eau virtuelle peuvent devenir un instrument pour remodeler l'allocation sectorielle des dotations hydriques. L'analyse du coût d'opportunité des usages céréaliers de l'eau, i.e. la valeur des usages alternatifs, est une composante clé des perspectives ouvertes par ce concept pour l'évaluation des choix entre production et recours au commerce international, et d'allocation intersectorielle de la ressource. Le commerce pourrait être utilisé pour préserver des ressources rares pour les affecter à des usages plus productifs et améliorer l'efficacité des usages. Des économies supplémentaires sur l'irrigation des céréales permettraient d'allouer l'eau disponible à des cultures à plus forte valeur ajoutée, et à d'autres secteurs comme l'industrie et le tourisme.

Ces deux problématiques s'imbriquent en Afrique du Nord où l'insuffisance de la ressource obligera à importer de plus en plus massivement d'eau virtuelle sous forme de céréales, mais pose en même temps la question de la place des productions domestiques, qui ne participent qu'à une part relativement faible et décroissante des besoins alimentaires, mais qui concentrent néanmoins une part importante de l'eau disponible. Si le commerce d'eau virtuelle a jusqu'à présent été utilisé comme variable d'ajustement palliatif aux déficits structurels et aux fluctuations interannuelles des productions alimentaires, il pourrait aussi devenir un outil de gestion de l'allocation des ressources hydriques.

L'analyse de l'opportunité d'une utilisation stratégique de cet instrument se concentre ici sur les substitutions intra-agricoles potentielles en Afrique du Nord. Les céréales sont souvent disponibles sur les marchés internationaux à des prix inférieurs aux coûts de production des pays caractérisés par des déficits hydriques structurels (El-Nasser, 2005). Il pourrait ainsi être rationnel de déplacer les usages de l'eau vers des cultures à haute valeur ajoutée, et d'augmenter les importations de céréales. Les choix de production ou d'importation ne dépendent cependant 
pas seulement des dotations hydriques absolues. L'analyse des avantages comparatifs des filières alternatives, nécessite d'intégrer toutes les ressources productives au-delà de l'eau, notamment les terres arables et le travail, et d'autres composantes de la fonction objectif que la gestion efficace de ce facteur, comme le besoin d'une croissance de l'économie et de l'emploi.

En Egypte, la stabilisation des importations et la croissance des productions céréalières sont le résultat d'investissements massifs dans l'irrigation et de programmes d'attribution de terres. Cette stratégie agricole et hydrique mais traduit cependant une forme de "maladaptation » à la rareté croissante de l'eau, également dommageable à l'emploi. L'analyse du contenu relatif en facteurs des cultures ${ }^{8}$ montre que la promotion des exportations de coton, de fruits et de légumes au détriment des exportations de riz et des cultures de blé et de maïs améliorerait les revenus, les opportunités d'emplois et la sécurité alimentaire, tout en permettant une utilisation plus efficace des ressources hydriques (Wichelns, 2001). Au Maghreb, l'analyse des usages alternatifs de la ressource doit distinguer les cultures pluviales et irriguées, pour cibler l'eau qui pourrait être réallouée. Dans le nord de la région, les contenus relatifs en eau et en travail montrent qu’il serait rationnel de substituer des cultures fruitières et maraîchères tournées vers l'exportation aux productions céréalières irriguées.

Une telle recomposition des filières agricoles, par le déplacement de productions céréalières affectées aux marchés domestiques vers des cultures d'exportation, et le remodelage des usages agricoles de l'eau qui en découle, augmenterait les exportations d'eau virtuelle. Elle améliorerait néanmoins l'efficacité de l'allocation de la ressource. Chaque $\mathrm{m}^{3}$ d'eau importé par la substitution de production de blé par des importations serait en effet acquis sur les marchés internationaux à un coût de 0,08 \$ en moyenne en Afrique du Nord. Réallouées par exemple aux cultures de tomates pour l'exportation, ces économies d'eau virtuelle permettraient de générer

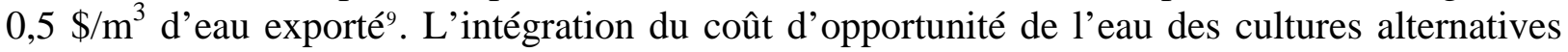
serait donc un outil pertinent d'adaptation à la rareté, qui participe simultanément à d'autres objectifs de développement comme l'emploi.

\subsection{Conditions et moyens de promotion de stratégies d'importation d'eau virtuelle}

Le commerce d'eau virtuelle vise à compenser les déficits hydriques locaux par un déplacement géographique de la production agricole et un déplacement sectoriel des usages domestiques de l'eau. En Afrique du Nord, l'importation des céréales couplée à la promotion de cultures à haute valeur ajoutée, et surtout la diversification de l'économie, serait une mesure holistique de gestion de la rareté de la ressource. Cependant, les conditions de promotion de cet instrument sont à analyser dans le contexte de la globalisation commerciale. La compatibilité relative avec le cadre de la GIRE, qui structure le renouveau des politiques de l'eau, est également un aspect majeur de l'opportunité et des risques d'une telle stratégie.

Plusieurs conditions sont nécessaires pour que le commerce d'eau virtuelle soit opportun. Le corollaire est que cet outil ne peut être mis en œuvre de manière radicale, mais seulement graduellement et plus ou moins partiellement, pour participer à la fois à la dimension environnementale, économique et sociale du développement durable. Il appelle donc un timing

\footnotetext{
${ }^{8}$ L'analyse du contenu relatif en facteurs des cultures en Egypte confirme la forte intensité en eau et en terre du blé, du maïs et surtout du riz, essentiellement tourné vers l'exportation. Le coton présente des besoins hydriques fortement inférieurs au riz, ainsi qu'un contenu en travail et une valeur ajoutée à l'hectare supérieurs aux cultures céréalières. La promotion des tomates améliorerait également les opportunités d'emploi à un coût hydrique raisonnable.

${ }^{9}$ Les calculs se fondent sur le prix moyen du blé importé et des tomates exportées par l'Algérie, l'Egypte et le Maroc entre 1990 et 2005 (FAO, TradeStat) et les DSE de ces cultures enregistrées dans ces pays. Les résultats montrent qu'un $\mathrm{m}^{3}$ d'eau importé sous forme de blé coûte en moyenne 0,02 \$ en Algérie, 0,05 \$ au Maroc et 0,16 \$ en Egypte, et qu'un $\mathrm{m}^{3}$ d'eau exporté sous forme de tomates génère respectivement $0,6,0,21$ et $0,71 \$$. L'utilisation stratégique du commerce d'eau virtuelle démultiplie donc la valeur des usages agricoles de l'eau.
} 
complexe entre le soutien des cultures céréalières et la réallocation progressive de l'eau vers d'autres secteurs.

Cette stratégie implique de pouvoir dégager les devises nécessaires à ces importations et s'adresse d'abord aux pays disposant de capacités d'exportation bien établies, comme les pays exportateurs de pétrole, ou capables de restructurer leurs secteurs d'exportation, notamment par la réallocation de l'eau. Les capacités d'absorption économique et sociale des effets du commerce d'eau virtuelle, en particulier une structure de l'économie qui offre des alternatives d'emploi et de revenus aux agriculteurs concurrencés par ces importations, constituent également un critère décisif pour l'introduction soutenable de cette stratégie (El-Nasser, 2005). Elle serait donc plus adaptée aux pays en voie d'industrialisation, à même de générer de nouvelles activités productives. Elle nécessite aussi des infrastructures de transport et un réseau de distribution de l'offre alimentaire adéquat en zone rurale. Dans la mesure où elle pourrait s'accompagner d'un processus de centralisation de l'offre alimentaire, elle augmente également le besoin d'une « bonne gouvernance ».

Etant donné l'importance écrasante de l'agriculture dans les structures socioéconomiques, les faibles capacités d'adaptation et l'insuffisance des infrastructures et des institutions, la promotion du commerce d'eau virtuelle ne serait probablement ni faisable ni désirable pour la plupart des pays d'Afrique sub-saharienne. Les conditions pourraient cependant être favorables dans les pays à revenus intermédiaires de la région MOAN (Allan, 2001).

En Afrique du Nord, l'importation des céréales et la promotion de cultures à haute valeur ajoutée seraient un ajustement stratégique de l'agriculture, même si à long terme la rareté de l'eau suggère que les réelles opportunités se situent en dehors de l'agriculture. D'autres pays méditerranéens, comme Israël, où les produits agricoles ne représentent qu'une faible part des échanges, ont déjà montré la possibilité d'une transition vers une économie de service intégrée à l'économie mondiale, couplée à l'importation des produits alimentaires et à la réallocation sectorielle de l'eau (Margat et Vallée, 1999).

Une importance sociopolitique majeure est assignée à l'agriculture dans la région, bien qu'elle nécessite d'importantes subventions. Le contexte politique est déterminant puisque, quelle que soit l'opportunité de cette stratégie, les céréales continueront à être produites domestiquement, autant que possible, tant que l'objectif d'autosuffisance alimentaire sera poursuivi. Les degrés de dépendance aux importations qui prévalent montrent cependant que l'enjeu de la sécurité alimentaire diffère clairement de celui de l'autosuffisance. Cet instrument ne concerne ainsi pas l'établissement d'un nouveau mécanisme, générateur d'une nouvelle dépendance, mais une augmentation incrémentale stratégique qui dégagerait des ressources hydriques déterminantes pour les autres secteurs (Turton, 1999).

Le commerce d'eau virtuelle s'intègre par définition dans un contexte de globalisation du commerce et forme ainsi une part importante des enjeux de la libéralisation des échanges agricoles. En Afrique du Nord, il consisterait d'abord à promouvoir une configuration des accords commerciaux et des partenariats qui facilite et sécurise l'offre alimentaire. Le rythme d'ouverture des marchés et la promotion de conditions favorables à la diversification des exportations sont également essentiels à l'analyse des relations entre libéralisation et commerce d'eau virtuelle.

La prise en compte des avantages comparatifs améliorerait l'emploi et la gestion de l'eau, mais la libéralisation mal maîtrisée du commerce agricole et l'ouverture trop rapide des marchés seraient néfastes au développement comme à l’environnement (Hervieu, 2006). La dualité de l'agriculture est un aspect sensible, puisque la libéralisation affecterait surtout la multitude des exploitations familiales des régions intérieures, essentiellement tournées vers 
l'autoconsommation, et pratiquant une agriculture pluviale avec peu d'usages alternatifs de la ressource. Elle pourrait simultanément intensifier les usages agricoles de l'eau des grands périmètres irrigués du nord de la région (SIA-EMFTA, 2004).

Les dotations factorielles relatives en eau ce constituent pas un élément structurant du commerce international puisque ce facteur n'est généralement pas rémunéré. L’intégration progressive du coût d'opportunité de l'eau, par la tarification des usages, est donc un préalable à l'ouverture des marchés. Et seule une stratégie d'ouverture progressive, dont le rythme serait conditionnel aux avancements dans la modification de la structure des incitations entre les filières alternatives, et qui accompagnerait les résultats de réallocation de l’eau des cultures céréalières irriguées vers d'autres usages, pourrait être compatible avec les objectifs qui sous-tendent le commerce d'eau virtuelle.

Ce remodelage sectoriel n'a cependant de sens que si les biens produits alternativement aux céréales sont commercialisables sur les marchés internationaux et si les conditions qui régissent le commerce sont favorables aux pays qui s'engageraient dans cette voie (Horlemann et Neubert, 2007). Dans le contexte actuel, l'augmentation et la diversification des exportations de produits agricoles et manufacturés ne peuvent progresser que lentement en Afrique du Nord, et les barrières non tarifaires des pays du Nord constituent un obstacle majeur à la pénétration des marchés internationaux ${ }^{10}$. Les stratégies d'eau virtuelle ne se révéleront fructueuses que si les accords commerciaux permettent préalablement d'alléger ces mesures protectionnistes, dommageables au développement durable de la région.

La concordance relative et les conflits potentiels avec les politiques de GIRE sont enfin un point de controverse majeur de l'opportunité du commerce d'eau virtuelle. Ce cadre d'action, institutionnalisé à Rio en 1992, met l'accent sur la gestion décentralisée de la demande comme moteur de l'adaptation à la rareté. Le commerce d'eau virtuelle est politiquement silencieux pour pallier la contradiction du principe d'autosuffisance alimentaire des pays contraints par les déficits hydriques. Le risque est qu'il devienne aussi une tentation forte pour retarder des réformes essentielles pour améliorer la soutenabilité des usages et remettre en cause des pratiques inadaptées. En Afrique du Nord, la gestion intégrée de la demande doit acquérir un rôle central pour cibler les causes institutionnelles et socioéconomiques des déficits hydriques.

Pour les politiques de l'eau, les importations d'eau virtuelle seraient dommageables en tant que stratégie commerciale séparée, qui se substituerait aux efforts de gestion de l'eau. Elles doivent donc s'intégrer à ce cadre d'action et compléter ces efforts pour être opportunes. Cet instrument serait compatible pour pallier les limites de la GIRE face aux déficits hydriques absolus ou en remplaçant des cultures intensives en eau dans les zones inadaptées. Il serait également synergique avec ce programme d'action, en rendant obsolètes des investissements insoutenables dans des infrastructures d'offre, comme certains projets de barrages et de transferts interrégionaux d'eau, ou d'utilisation à grande échelle de ressources non renouvelables. L'intégration du commerce d'eau virtuelle dans le cadre de la GIRE ne sera aussi possible qu'en adoptant une approche décentralisée de cet instrument, fondée sur l'intégration progressive du coût d'opportunité de l'eau dans les décisions d'usages plutôt que sur des restrictions sectorielles de l'accès à la ressource décidées centralement (Hummel, 2005).

\section{Conclusion}

\footnotetext{
${ }^{10}$ Le Maroc a conclu en 2004 un accord de libre échange avec les USA. II a obtenu le quasi libre accès pour la totalité de ses produits d'exportation et s'est engagé en contrepartie sur un calendrier d'ouverture aux produits américains. Mais, l'asymétrie domine puisque si les USA ont obtenu des possibilités inédites d'accès aux marchés marocains, le Maroc ne paraît pas en mesure de tirer réellement avantage des opportunités qui lui sont offertes car il butte sur d'importantes barrières non tarifaires (conditions sanitaires et phytosanitaires, circuits de commercialisation, taille critique)
} 
Le commerce d'eau virtuelle est un concept d'analyse pertinent des enjeux liant les stratégies d'adaptation à la rareté croissante de l'eau aux réflexions sur l'opportunité et les risques de la globalisation. Au-delà de la dimension incompressible des importations céréalières et de l'utilisation ad hoc du commerce d'eau virtuelle, l'utilisation stratégique de cet instrument pour remodeler l'allocation sectorielle de l'eau pourrait être une mesure holistique d'adaptation à la rareté, alternative aux tentatives d'intensification de l'irrigation des céréales. En Afrique du Nord les conditions socioéconomiques pourraient être propices, mais la compatibilité relative avec la libéralisation commerciale reste tributaire du rythme de l'ouverture des marchés domestiques, comparé aux avancements dans la restructuration des productions et dans l'allègement des barrières commerciales des pays développés. Les pays industrialisés qui ont reconnu leur responsabilité dans le changement climatique, et qui se sont engagés à promouvoir l'adaptation et à participer à son financement dans les PVD, ont donc un rôle important pour promouvoir une configuration des accords commerciaux favorable à une transition vers des économies moins sensibles aux disponibilités hydriques et intégrées à l'économie mondiale en Afrique du Nord.

Agoumi A., Senoussi S., et al., 1999, Changements climatiques et ressources en eau, Hydroécologie appliquée, 12(11), pp. 163-182

Allan J.A., 1996, The political economy of water: reasons for optimism but long term caution, In: Allan J.A. (ed.), Water, Peace and the Middle East: Negotiating resources in the Jordan basin, London

Allan J.A., 2001, Virtual water - Economically invisible and politically silent: A way to solve strategic water problems, International Water and Irrigation Journal, 21(4), pp. 39-41

Benblidia M., Margat J., Vallée D., 1997, L’eau en région méditerranéenne. Situations, perspectives et stratégies pour une gestion durable de la ressource, Communication à la conférence euro-méditerranéenne sur la gestion de l'eau, Marseille, 25-26 novembre, Plan Bleu

Bindi M., Moriondo M., 2005, Impact of a $2^{\circ} \mathrm{C}$ global temperature rise on the Mediterranean region: Agriculture analysis assessment, In : C. Giannakopoulos, M, Bindi, M. Moriondo, et al., Climate change impacts in the Mediterranean resulting from a $2^{\circ} \mathrm{C}$ global temperature rise, WWF Report, pp. 54-66

Boko, M., Niang I., Nyong A., et al., 2007, Africa. In : IPCC, Climate Change 2007: Impacts, Adaptation and Vulnerability. Contribution of Working Group II to the Fourth Assessment Report of the IPCC, pp. 433-467

El-Nasser H., 2005, Virtual water in the MENA region, Expert statement for the research project « Virtual Water A realistic concept for resolving the water crises? », German Development Institute

FAO, AQUASTAT, base de données en ligne, http://www.fao.org/nr/water/aquastat/data/query/index.html

FAO, TradeSTAT, base de données en ligne, http://faostat.fao.org/site/535/default.aspx

Hervieu B., 2006, L’agriculture est un secteur stratégique en Méditerranée, Notes d’Analyse du CIHEAM, 18, 8 p.

Hummel D., 2005, Water and food security: What are the demographic, institutional and socio-cultural conditions for virtual water trade?, Expert statement for the research project « Virtual Water - A realistic concept for resolving the water crises ? », German Development Institute

Hoekstra A., Hung P., 2002, Virtual water trade: A quantification of virtual water flows between nations in relation to international crop trade, Value of Water Research Report Series, 11, IHE Delft, 66 p.

Horlemann L., Neubert S., 2007, Virtual water trade: A realistic concept for resolving water crisis?, German Development Institute Studies, 119 p.

Hulme M., Wigley T., et al., 2000, Using a Climate Scenario Generator for Vulnerability and Adaptation Assessments: MAGICC and SCENGEN Version 2.4, Norwich

Katz R., Brown B.J., 1992, Extreme events in a changing climate, Climatic Change, 21(3), pp. 289-302

Margat J., Vallée D., 1999, Vision méditerranéenne sur l'eau, la population et l'environnement au XXI siècle, Plan Bleu, 83 p.

Ministère de l'Aménagement du territoire et de l'environnement. Algérie, 2001, Elaboration de la stratégie et du plan d'action national des changements climatiques. Communication nationale à la CCNUCC, 155 p. 
Nakicenovic N., Alcamo J., et al., 2000, Emissions scenarios, Special Report of Working Group III of the IPCC, $570 \mathrm{p}$.

PNUD-FEM, 1998, Changements climatiques et ressources en eau dans les pays du Maghreb, Algérie - Maroc Tunisie, Enjeux et perspectives

Renault D., 2003, Value of virtual water in food : Principles and virtues, In : Hoekstra A.Y. (eds), Virtual Water Trade - Proceedings of the international expert meeting on virtual water trade, Value of water research report series, 12, IHE Delft, pp. 77-91

Senouci S., 2002, Changement climatique et ressources en eau dans le bassin versant de l'Ouergha, Thèse de doctorat, Université Hassan II, Casablanca

SIA-EMFTA, 2004, Sustainability impact assessment study of the Euro-Mediterranean free trade area, Institute for Development Policy and Management, University of Manchester, 115 p.

Turton A., 1999, Precipitation, people, pipelines and power: Toward a “virtual water" based political ecology discourse, MEWREW occasional paper, London

Wichelns D., 2001, The role of "virtual water" in efforts to achieve food security and other national goals, with an exemple from Egypt, Agricultural Water Management, 49(2), pp. 131-151

Yang H., Zehnder A., 2002, Water scarcity and food import: A case study for southern Mediterranean countries, World Development, 30(8), pp. 1413-1430

Yang H., Reichert P., et al., 2003, A water resources threshold and its implications for food security, In : Hoekstra A.Y. (ed), Virtual Water Trade - Proceedings of the international expert meeting on virtual water trade, Value of water research report series, 12, IHE Delft, pp. 111-117 